\title{
Toward Automatic Computer Aided Dental X-ray Analysis Using Level Set Method
}

\author{
Shuo $\mathrm{Li}^{1}$, Thomas Fevens ${ }^{1}$, Adam Krzyżak ${ }^{1}$, Chao Jin ${ }^{1}$, and Song $\mathrm{Li}^{2}$ \\ 1 Medical Imaging Group, \\ Department of Software Engineering and Computer Science, \\ Concordia University, Montréal, Québec, Canada \\ \{shuo_li, fevens, krzyzak, chao_jin\}@cs.concordia.ca \\ 2 School of Stomatology, Anhui Medical University, Hefei, Anhui, P. R. China \\ xlisong@sohu.com
}

\begin{abstract}
A Computer Aided Dental X-rays Analysis (CADXA) framework is proposed to semi-automatically detect areas of bone loss and root decay in digital dental X-rays. In this framework, first, a new proposed competitive coupled level set method is proposed to segment the image into three pathologically meaningful regions using two coupled level set functions. Tailored for the dental clinical environment, the segmentation stage uses a trained support vector machine (SVM) classifier to provide initial contours. Then, based on the segmentation results, an analysis scheme is applied. First, the scheme builds an uncertainty map from which those areas with bone loss will be automatically detected. Secondly, the scheme employs a method based on the SVM and the average intensity profile to isolate the teeth and detect root decay. Experimental results show that our proposed framework is able to automatically detect the areas of bone loss and, when given the orientation of the teeth, it is able to automatically detect the root decay with a seriousness level marked for diagnosis.
\end{abstract}

\section{Introduction}

The past few years has seen a great increase in the usage of digital dental X-rays in dental practices in North America. Their adoption is motivated by the fact that digital X-rays systems are more sensitive than X-ray film systems while allowing for up to a $90 \%$ reduction in a patient's exposure to X-rays compared to traditional systems. With the higher resolution digital X-rays of sections of the teeth, or of the entire jaw, comes the possibility of more accurate diagnoses.

Dental X-rays play an important role in detecting such pathological problems such as periodontitis, chronic periapical periodontitis and bone loss that cannot be seen during a visual examination. Periodontitis is a dental disorder that results from the progression of gingivitis, involving the inflammation and infection of the ligaments and bones that support the teeth. Early detection of bone loss and root decay is very important since often they can be remedied by dental procedures, such as a root canal, for example. Without early treatment, bone loss may lead to loss of teeth or erosion of the jaw bone. In the dental X-ray film 
based environment, the dentist does the analysis of the X-ray in their head. With the increasingly wide use of digital radiography, the dental X-rays are accessible electronically which makes computer aided dental X-rays analysis possible.

However, although dental X-rays are widely used, it is a challenging task to do automatic, or even semi-automatic, computer aided dental X-rays analysis. Compared with other types of images, dental X-rays analysis is a challenging problem for classical image processing methods due to the following characteristics: (1) poor image modalities: noise, low contrast, and sample artifacts; (2) very complicated topology; and (3) there may not be clear lines of demarcation between regions of interest, which is especially true for dental X-rays since problem teeth tend to have very complicated structures and are normally coupled with healthy teeth. Therefore dental X-rays are normally inspected by a dentist. Although efficient, human inspection requires specialized training which is increasingly expensive. In addition, human inspection gives a subjective judgment which may vary from person to person, and, as such, does not give a quantitative measurement. Inspection results could be affected by many factors, such as fatigue and distraction by other features in the image, for example. Also, some early bone loss may not be visible to the human eye. Early detection of bone loss and root decay is very important since often they can be remedied by dental procedures, such as a root canal, for example. Without early treatment, bone loss may lead to loss of teeth or erosion of the jaw bone. All these issues indicate a need for effective automatic dental X-rays analysis.

In this paper, we report on innovative work on computer aided dental X-rays analysis which semi-automatically provides indications to the dentist aid in finding bone loss and root decay, which are the primary reasons that X-rays are taken in many countries. For this implementation, we will be dealing primarily with X-rays which are close-up views of a few individual teeth, a common type of dental X-rays, although the approach developed here can be adapted to larger scale dental X-rays. Compared with panoramic dental X-rays which include the entire jaw region, closeup images taken for these purposes are more challenging since the orientation of the teeth may not be fixed and problem areas are either complicated, or easily overlooked. To the best of our knowledge, we are the first group working towards automatic computer aided dental X-rays diagnosis for the detection of bone loss and root decay. This paper reports on our preliminary results towards this goal.

\section{Proposed Framework}

As shown in Fig. 1, the framework consists of two phases: segmentation and analysis. First, we employ a new proposed competitive level set segmentation method to segment the image into three regions using two coupled level set functions. Based on the segmentation results, an analysis scheme is applied. The scheme first builds an uncertainty map which is then used to automatically mark any areas of bone loss. Subsequently, an average intensity profile based method is employed to isolate the teeth and detect possible root decay. Finally the estimated seriousness level of the root decay will be marked. 


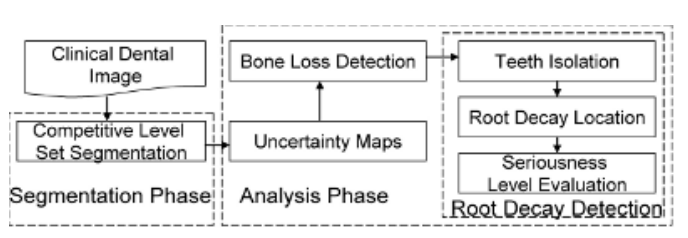

Fig. 1. Framework diagram

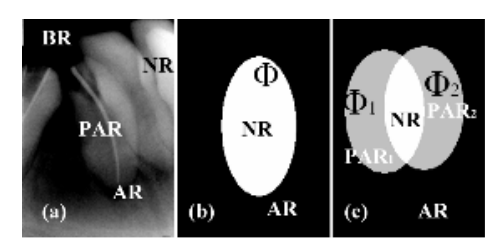

Fig. 2. Region modelling of dental image (a), one level set segmentation

(b) and competitive level set (c)

\subsection{Variational Level Set Segmentation}

In this implementation, we propose a new variational level set segmentation method driven by pathological energy modelling. The modelling explicitly incorporates regions of problems as part of the modelling, so the identification of such areas would be an automatic product of the segmentation. The level set method segments the dental X-rays into three regions: Normal Region (NR), Potential Abnormal Region (PAR), Abnormal and Background Region (ABR) using two competitive level set functions.

Competitive Level Set Segmentation. With an evolving curve $C$, one level set function divides the image $(u)$ into two parts: Normal Region $\Omega_{N R}$ ("+" region) and Abnormal Region $\Omega_{A R}$ ("-" region) as shown in Fig. 2(b). The energy functional is given by

$$
E(\Phi)=\beta_{1} \int_{\Omega_{N R}} \frac{\left(u-c_{N R}\right)^{2}}{\sigma_{N R}^{2}} d x d y+\beta_{2} \int_{\Omega_{A R}} \frac{\left(u-c_{A R}\right)^{2}}{\sigma_{A R}^{2}} d x d y,
$$

where $c_{i}$ is the mean grey value of the $\Omega_{i}, \sigma_{i}$ is the variance and $\beta_{i}$ is a constant.

However for the diagnosis of pathological problems, potential problems areas which might be between normal and abnormal regions are of particular interest. Therefore we propose a competitive coupled level set model for two level set functions to segment the image into three regions. As shown in the Fig. 2(a), a X-ray image $\left(u_{0}\right)$ can be divided into four regions of interest: the Normal Region $\left(\Omega_{N R}\right)$, the Potential Abnormal Region $\left(\Omega_{P A R}\right)$, the Abnormal Region $\left(\Omega_{A R}\right)$ and the Background Region $\left(\Omega_{B R}\right)$. Since $\Omega_{A R}$ and $\Omega_{B R}$ is not separable in terms of intensity values, so in the segmentation, we take $\Omega_{A R}$ and $\Omega_{B R}$ to be a single region: the Abnormal and Background Region $\left(\Omega_{A B R}\right)$. The energy functional for the two coupled level set functions $\left(\Phi_{1}\right.$ and $\left.\Phi_{2}\right)$ can be modeled as:

$$
\begin{gathered}
E\left(\Phi_{1}, \Phi_{2}\right)=\lambda_{1} \int_{\Omega_{N R}} \frac{\left(u-c_{N R}\right)^{2}}{\sigma_{N R}^{2}} d x d y+\lambda_{3} \int_{\Omega_{A B R}} \frac{\left(u-c_{A B R}\right)^{2}}{\sigma_{A B R}^{2}} d x d y \\
+\lambda_{2} \int_{\Omega_{P A R}} \operatorname{Min}\left(\frac{\left(u-c_{P A R_{1}}\right)^{2}}{\sigma_{P A R_{1}}^{2}}, \frac{\left(u-c_{P A R_{2}}\right)^{2}}{\sigma_{P A R_{2}}^{2}}\right) d x d y
\end{gathered}
$$

where the function $\operatorname{Min}(x, y)$ returns the smaller value of $x$ and $y$, and $\lambda_{i}$ is a constant. The modelling is in the same spirit as multiphase modelling in [1].

Using competitive coupled level set functions, if both level set functions classify an area as a normal region, we take it as a normal region $\Omega_{N R}$; if both level 
set functions classify a region as an abnormal and background region, we take it as an abnormal and background region $\Omega_{A B R}$; however if only one of the level set functions segments a region as a normal region, the region will be taken as a potential abnormal region $\Omega_{P A R}$. Compared to three coupled level set functions, two coupled level sets is able to achieve faster and more robust segmentation as described in [1.

To achieve fast and robust segmentation, a hybrid coupled level sets functional that combines minimal variance (Eq. 2), the optimal edge integrator [2] and the geodesic active contour model [3] is used:

$$
E=E\left(\Phi_{1}, \Phi_{2}\right)-\gamma_{1} E_{L A P}+\gamma_{2} E_{G A C}
$$

where $\gamma_{i}$ are constants.

The geodesic active contour $\left(E_{G A C}\right)$ and edge functional $\left(E_{L A P}\right)$ are defined in Eq. 4. The edge functional was proposed in [2] where the authors show that a Laplacian edge detector $\Delta u$ provides optimal edge integration with regards to a very natural geometric functional.

$$
\begin{gathered}
E_{G A C}(C)=\iint g(C) d x d y \\
E_{L A P}(C)=\int_{C}<\nabla, \mathbf{n}>d s+\iint_{\Omega_{C}} K_{u}|\nabla u| d x d y .
\end{gathered}
$$

Here $K_{u}$ is the mean curvature of the level set function, $\mathbf{n}$ is the unit vector normal to the curve and $d s$ is the arc length of curve $C$. Function $g(x, y)$ is an inverse edge indicator function introduced in [4, and defined as $g(x, y)=$ $\alpha^{2} /\left(\alpha^{2}+|\nabla u|^{2}\right)$, where $\alpha$ is a constant and $\nabla$ is the gradient operator. The level set functions $\Phi_{i}$ are derived from the functional in Eq. 3 as shown:

$$
\begin{gathered}
\frac{\partial \Phi_{1}}{\partial t}=\delta_{\varepsilon}\left(\Phi_{1}\right)\left[\gamma_{2} \operatorname{div}\left(g \frac{\nabla \Phi_{1}}{\left|\nabla \Phi_{1}\right|}\right)-\frac{\left(u-c_{N R}\right)^{2}}{\sigma_{N R}^{2}} H\left(\Phi_{2}\right)-\frac{\left(u-c_{P A R}\right)^{2}}{\sigma_{P A R}^{2}}\left(1-2 H\left(\Phi_{2}\right)\right)(5)\right. \\
\left.+\frac{\left(u-c_{A B R}\right)^{2}}{\sigma_{A B R}^{2}}\left(1-H\left(\Phi_{2}\right)\right)-\gamma_{1} u_{\xi \xi}\right], \\
\frac{\partial \Phi_{2}}{\partial t}=\delta_{\varepsilon}\left(\Phi_{2}\right)\left[\gamma_{2} \operatorname{div}\left(g \frac{\nabla \Phi_{2}}{\left|\nabla \Phi_{2}\right|}\right)-\frac{\left(u-c_{N R}\right)^{2}}{\sigma_{N R}^{2}} H\left(\Phi_{1}\right)+\frac{2\left(u-c_{P A R}\right)^{2}}{\sigma_{P A R}^{2}} H\left(\Phi_{1}\right)\right. \\
\left.+\frac{\left(u-c_{A B R}\right)^{2}}{\sigma_{A B R}^{2}}\left(1-H\left(\Phi_{1}\right)\right)-\gamma_{1} u_{\xi \xi}\right] .
\end{gathered}
$$

Here, $H(\cdot)$ is the Heaviside function, $\operatorname{div}(\cdot)$ is the divergence operator, and $u_{\xi \xi}=$ $\Delta u-K_{u}|\nabla u|$.

Although we only apply competitive level set segmentation to dental X-rays, the segmentation method can be extended to X-rays and some types of CT images for three region segmentation using two level set functions.

Segmentation Phase. To apply the level set method in the clinical environment, we adapt the segmentation framework proposed by Li et al. 4, 5, which uses a trained SVM to provide a good initial contour for the level set method which greatly speeds up convergence of the coupled level set functions. Following the same principle, we use an SVM to provide initial contours for two coupled 
level set functions. The purpose is not only to speed up segmentation convergence, but also to use competitive couple level set functions to find regions of interest, as discussed in section 2.1. The segmentation phase has two stages: a training stage and a clinical segmentation stage.

During the training stage, manually chosen representative images are segmented by hierarchical level set region detection using the Chan and Vese level set method [6]. In the hierarchical level set region detection, first a level set function is used to separate $\Omega_{A B R}$ from the rest of the image $\left(\Omega_{A R}\right.$ and $\left.\Omega_{B R}\right)$. Then another level set function is used to separate $\Omega_{A R}$ and $\Omega_{B R}$. Then these results are used to train an SVM classifier.

During the clinical segmentation stage, dental X-rays are first classified by the trained SVM. The classifier is able to classify three regions $\left(\Omega_{A B R}, \Omega_{N R}\right.$ and $\left.\Omega_{P A R}\right)$ based only on intensity which may not be accurate. Then two coupled level set functions are used to further segment the images. For $\Phi_{1}$, we set classified $\Omega_{N R}$ as the "+" region and rest of the image as the "- " region; for $\Phi_{2}$, we set classified $\Omega_{N R}$ and $\Omega_{P A R}$ region as the "+" region and rest of the image as the "-" region. Although SVM is only able to give a coarse segmentation, it provides a very good and competitive initial contours for two coupled level set functions. The final segmentation will be obtained by evolution of these two level set curves.

\subsection{Analysis Phase}

The analysis phase contains three steps: uncertainty map building, bone loss detection and root decay detection. The first two are fully automatic. The only manual input is the image orientation during root decay detection. This requirement is not difficult to accommodate in the dental clinical environment.

Uncertainty Maps. First for each image, an uncertainty map is built based on following uncertainty measurement:

$$
\psi=\frac{\left(u-c_{N R}\right) \tau_{1}+\left(u-c_{P A R}\right)\left(\tau_{2}-2 \tau_{1}\right)+\left(u-c_{A B R}\right)\left(1-H\left(\phi_{1}\right)\right)\left(1-H\left(\phi_{2}\right)\right)}{\sigma_{N R} \tau_{1}+\sigma_{P A R}\left(\tau_{2}-2 \tau_{1}\right)+\sigma_{A B R}\left(1-H\left(\phi_{1}\right)\right)\left(1-H\left(\phi_{2}\right)\right)}
$$

where $\tau_{1}=H\left(\phi_{1}\right) H\left(\phi_{2}\right)$ and $\tau_{2}=H\left(\phi_{1}\right)+H\left(\phi_{2}\right)$.

Bone Loss Detection. Areas of bone loss will generally occur in those regions of high uncertainty. Therefore, we mark these areas with different levels of emphasis according to the uncertainty measurement and region segmented.

Although the uncertainty map is an objective uncertainty measure, it fails to provide direct visual cues. To achieve the visual assistance, the RGB channels of the image are used to couple the intensity values of the image with the degree of uncertainty at each pixel. For all regions, the $\mathrm{G}$ channel is used to represent the intensity value of each pixel of the original dental X-ray image. The uncertainty values are nonlinearly (For this implementation, users can interactively choose the polynomial functions to get best visualization results.) scaled to the range -255 to 255 . To differentiate between the three regions, we apply the following schemes to $\mathrm{R}$ and $\mathrm{B}$ channel for pixels in each region: 1) For $\Omega_{A B R}, \mathrm{~B}$ channel is 


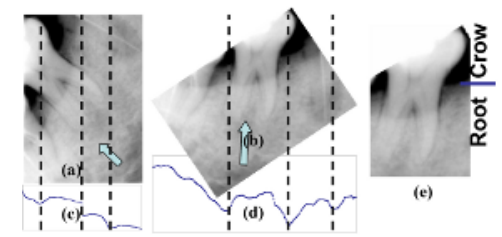

Fig. 3. Teeth isolation. (a) Original image. (b) Rotated image. (c) Integrated intensity. (d) Average intensity profile. (e) Two parts of a tooth: crown and root.

set to 0 . The $\mathrm{R}$ channel is set to the uncertainty value if it is negative; otherwise the $\mathrm{R}$ channel is set to 0.2$)$ For $\Omega_{P A R}$, both the $\mathrm{R}$ and $\mathrm{B}$ channels are set to 200 to emphasize this region. 3) For $\Omega_{N R}$, both the $\mathrm{R}$ and $\mathrm{B}$ channels are set to the uncertainty value if it is negative; otherwise, both channels are set to 0 .

Root Decay Detection. Root decay detection consists of three steps: tooth isolation, root decay location and seriousness level evaluation. This is a semiautomatic process in which the orientation of the teeth is supplied manually.

Teeth isolation: As suggested by Jain et al. [7, since the teeth usually yield higher intensity values than the jaws and other tissues, the gap of teeth will have a very low value on the integrated intensity value profile which are the sums of the intensities of pixels along the vertical direction. However, unlike dental X-ray images used for a forensic purpose which can be assumed to have certain orientation, clinical dental X-rays used to detect root decay, etc., could have any orientation. As shown in the Fig. 3(a) and (c), if the orientation varies, this profile method will not able to obtain the correct isolation. Therefore as a preliminary step, we assume that the orientation is given. Then we rotate the image according to the given orientation so that the teeth are aligned in a consistent direction. After rotation, instead of using an integrated intensity value, we use the average of the intensity value (the integrated intensity value divided by the number of pixels) as shown in Fig. 3(b) and (d). After rotation, we use an SVM to judge where are the gaps in the teeth using a $1 \mathrm{D}$ window based feature vector from average intensity profile for training and classification.

Root decay location and seriousness level evaluation: Three types of regions are considered to be root decay if they are found at the root of teeth with the following seriousness levels, in order of most serious to least serious: 1 . advise level: if the $\Omega_{A B R}$ is found at the root of the tooth; 2 . warning level: if the $\Omega_{P A R}$ of any uncertainty is found at the root of the tooth; 3 . attention level: if a high uncertainty area of $\Omega_{N R}$ is found at the root of the tooth.

\section{Experimental Results}

\subsection{Segmentation}

Figs. 45] and [6] show results of competitive level set segmentation. Since regions of problems are incorporated as part of the modelling, the identification of 

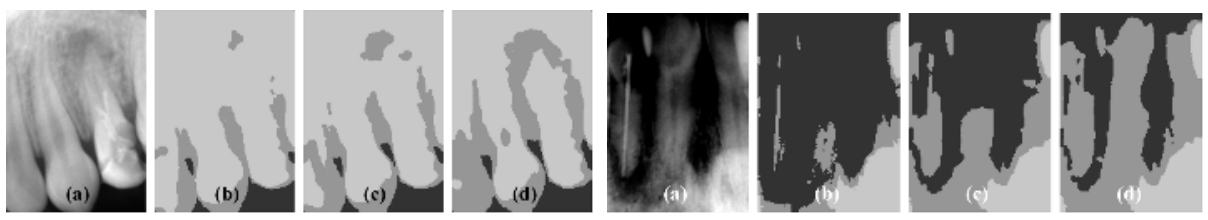

Fig. 4. Segmentation Results. (a) Original Fig. 5. Segmentation Results. (a) Original image. (b) Initial condition provided by image. (b) Iteration 0 provided by SVM. (c) SVM. (c) Iteration 20. (d) Iteration 60. Iteration 40. (d) Iteration 80.

such areas would be an automatic product of the segmentation. The pathological meaningful segmentation can be used at the next stage of analysis. In additional, as shown in Fig. 4 and 5, although the SVM only gives a rough approximate segmentation, it is able to provide a good competitive initial contour for two level set functions, $\Phi_{1}$ and $\Phi_{2}$, which accelerates the segmentation. Indeed, the competitive level set segmentation is robust to the placement of the initial contours so that even when the initial contour does not closely correspond to the final segmentation, the level set functions can still achieve an accurate segmentation as shown in Fig. 5 .

\subsection{Analysis}

Based on the uncertainty map and segmentation results, the analysis is able to indicated some possible areas in the image as shown in Fig. 6(c) in which those bone loss areas are emphasized by the color channel scheme. The scheme provides more direct visual cues which will greatly reduce the possibility that those areas, the area pointed to by an arrow in Fig. 66(c) for example, might be overlooked.

Figs. 6. 7)and 8 show results of teeth root decay detection. In these examples, the segmentation and uncertainty map successfully locate the area of bone loss and, after being given the orientation of the image, the area of root decay can be automatically detected. Fig. [6 shows an interesting result of automatic root decay detection. In this example, the system automatically detected three root
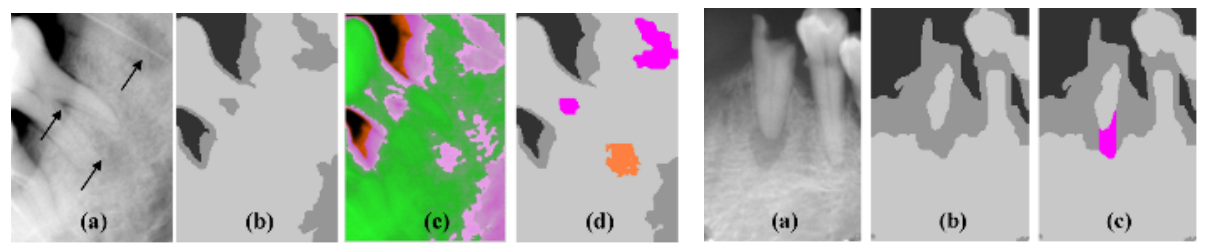

Fig. 6. Segmentation Results. (a) Original im- Fig. 7. Root Decay Detection Reage. (b) Segmentation results. (c) Bone loss area sults. (a) Segmentation Results. (b) marked with color channel method. (d) Root de- Root decay detected. cay detected. 


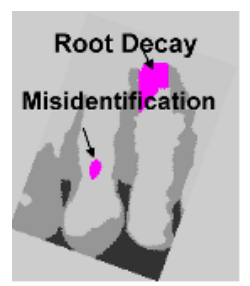

Fig. 8. Root decay detection of Fig. 6
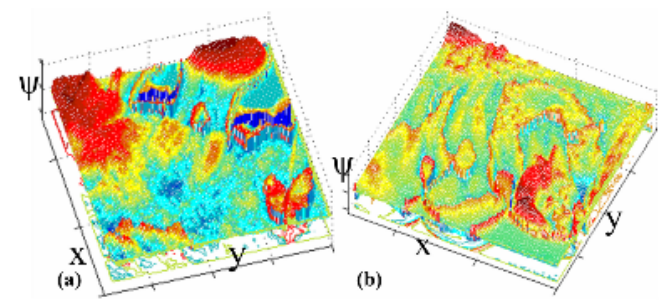

Fig. 9. Uncertainty maps of (a) Fig. 4 and (b) Fig. 6

problems with the orientation given. Two of them are warning level (pink area) and one is attention level (orange area) as shown in Fig. 6(d). Fig. 9 shows two of resulting uncertainty maps. The map is able to give a general idea for the problem areas. And more importantly, it provides a digitized uncertainty measurement. Altogether 50 dental X-rays with different levels of bone loss and root decay are used to test the proposed framework and the results validated by dentist. The experimental results show that the proposed framework is able to help to find all the areas of bone loss. For the root decay detection, the proposed framework is able to find all of them. But due to complexity of the dental X-rays, there are still 4 misidentifications of root decay. One of these misidentifications are indicated by arrows in Fig. 8

\section{Summary and Conclusions}

Leveraging the transition towards a totally electronic format for dental X-rays, a framework to semi-automatically detect areas of bone loss and root decay in these images is proposed. The system is designed in particular for the dental clinical environment through the use of a classifier to ensure accurate segmentation of these images. Utilizing these segmentations and a computed uncertainty map, regions of pathological abnormality are emphasized for the dentist's attention. Experimental results indicate that the system correctly identifies such problem areas with a few misidentifications. To the best of our knowledge, this is the first work on semi-automatic dental X-ray computer aided diagnosis.

\section{References}

1. L. Vese and T. Chan, "A multiphase level set framework for image segmentation using the mumford and shah model," International Journal of Computer Vision, vol. 50, no. 3, pp. 271-293, 2002.

2. R. Kimmel and A. M. Bruckstein, "Regularized Laplacian zero crossings as optimal edge integrators," International Journal of Computer Vision, vol. 53, pp. 225-243, July 2003.

3. V. Caselles, R. Kimmel, and G. Sapiro, "Geodesic active contours," International Journal of Computer Vision, vol. 22, pp. 61-79, 1997. 
4. S. Li, T. Fevens, and A. Krzyżak, "Image segmentation adapted for clinical settings by combining pattern classification and level sets," in Medical Image Computing and Computer-Assisted Intervention (MICCAI), (St-Malo, France), pp. 160-167, 2004.

5. S. Li, T. Fevens, and A. Krzyżak, "An SVM based framework for autonomous volumetric medical image segmentation using hierarchical and coupled level sets," in Computer Aided Radiology and Surgery (CARS), (Chicago, USA), pp. 207-212, 2004.

6. T. Chan and L. Vese, "Active contour model without edges," IEEE Trans. on Image Processing, vol. 24, pp. 266-277, 2001.

7. A. K. Jain and H. Chen, "Matching of dental x-ray images for human identification," Pattern Recognition, vol. 37, no. 7, pp. 1519-1532, 2004. 